\title{
Talc Pleurodesis Through Very-Small-Bore Catheters in Patients with Recurrent Malignant Pleural Effusion
}

\author{
Ali Özgen
}

Department of Radiology, Yeditepe University School of Medicine, İstanbul, Turkey

\begin{abstract}
Objective: Malignant pleural effusion (MPE) is a frequent and disturbing complication of metastatic disease. Talc pleurodesis via percutaneosly placed $12-18 \mathrm{~F}$ catheters is an effective procedure to treat recurrent MPE. We aimed to determine the efficiency of talc pleurodesis through very-small-bore catheters in the treatment of recurrent malignant pleural effusion.
\end{abstract}

Methods: We performed 13 talc pleurodesis procedures in 10 patients with recurrent MPE via pre-existing 7F (6 patients) and $8 \mathrm{~F}$ (4 patients) pig-tail catheters. We analyzed technical and clinical success of the procedure.

Results: All procedures were performed successfully. Complete or partial clinical success was achieved in 8 out of 10 patients. No major complication was observed.

Conclusion: Talc pleurodesis through 7F or 8F catheters may be performed in selected patients with reduced patient discomfort, and similar success rates that was obtained using higher caliber catheters in the treatment of recurrent MPE.

Keywords: Fluoroscopy, malignant, pleural effusion, pleurodesis, talc, therapy

\section{INTRODUCTION}

Malignant pleural effusion (MPE) is a frequent and disturbing complication of metastatic disease $(1,2)$. Talc pleurodesis is an effective procedure to treat recurrent MPE (3-7). Intrapleural administration of talc can be performed via videothoracoscopy or a chest tube, and this requires hospital admission $(6,7)$. Talc pleurodesis can also be performed as an outpatient procedure via percutaneously placed $12-18 \mathrm{~F}$ catheters $(4,5,8)$. The objective of this study was to present results of 13 talc pleurodesis procedures performed in 10 patients using $7 \mathrm{~F}$ or $8 \mathrm{~F}$ catheters, which, to the best of our knowledge, has not been presented in the literature before.

\section{METHODS}

\section{Subjects}

This study was approved by the Ethics Committee of our institution. Between September 2011 and December 2014, we performed 13 talc pleurodesis procedures in 10 patients with metastatic disease via pre-existing 7F (6 patients) and $8 \mathrm{~F}$ ( 4 patients) pig-tail catheters. All patients were referred from the department of oncology with the diagnosis of recurrent MPE. One patient had metastatic mesothelioma, 4 had lung cancer, and 5 had metastatic breast cancer. All patients suffered from moderate to severe dyspnea before the procedure. Four patients were treated as outpatients, while 6 patients had been hospitalized during the procedure because of the systemic treatment they had received. The expected survival time was more than 3 months for all patients. 


\section{Pleural Catheter Placement}

All patients were examined with ultrasound prior to pleural catheter placement. If the pleural fluid was anechoic or hypoechoic and has no septations, we aspirated a small amount using a $22 \mathrm{G}$ needle to see whether the fluid was hemorrhagic and/or dense. When we could easily aspirate a non-hemorrhagic fluid without any apparent septations and echoes on ultrasound, we inserted a 7F or 8F pig-tail catheter. Otherwise, we used a $12 \mathrm{~F}$ or $14 \mathrm{~F}$ pig-tail catheter with larger holes to treat such complicated pleural effusions. Patients with complicated pleural effusions were not considered as candidates for talc pleurodesis. All procedures were performed with real-time ultrasound guidance under local anesthesia with patients in a sitting position by an interventional radiologist. Informed consent was obtained from all patients.

\section{Talc Pleurodesis}

All procedures were performed via pre-existing catheters when the daily drainage of pleural fluid was less than $100 \mathrm{~mL}$. The patient was instructed to lie in a supine position on the fluoroscopy table. The location of the catheter was checked under fluoroscopy by opening the stopcock and observing free pleural fluid drainage (Figure 1a). If free fluid drainage was not observed, $10-20 \mathrm{~mL}$ of iodinated contrast medium was given under flouroscopic guidance to confirm the location of the catheter (Figure $1 \mathrm{~b}$ ). Then, $10 \mathrm{~mL}$ of $2 \%$ lidocain was given via the catheter into the pleural space. Patients were instructed to lie in supine and prone positions each for $10 \mathrm{~min}$ for lidocain to disperse evenly. Then, $4 \mathrm{~g}$ of sterile talc (Steritalc, Novatech, France) with an average particle size of $25 \mu \mathrm{m}$ in $50 \mathrm{~mL}$ of $0.9 \% \mathrm{NaCl}$ solution was given via the catheter. Intravenous analgesia was given by an anesthesiologist only when necessary. Stopcock was then closed after flashing the catheter with $5 \mathrm{~mL}$ of $0.9 \% \mathrm{NaCl}$ solution. A control standard chest X-ray was obtained (Figure 1c). Patients were then instructed to lie supine, on the right side, prone, and on the left side each for 15 minutes for a total of 2 hours. Then, the stopcock was opened for free fluid drainage. Catheters were flushed with $10 \mathrm{~mL}$ of sterile saline twice daily. Once the drainage stopped, the catheter was removed.

\section{Follow-up and Assessment of Efficacy}

Patients were followed by the departments of oncology, chest diseases, and radiology with standard chest X-rays to determine the effectiveness of the pleurodesis, while they were routinely followed clinically. The efficacy of talc pleurodesis was defined in 3 categories during the follow-up: complete (none or minimal pleural fluid accumulation), partial (relapse of some fluid that did not require treatment), and failure (relapse of fluid that required further intervention). If pleurodesis failed, the procedure was repeated one more time with the same technique.

\section{RESULTS}

All procedures were performed easily as a single-step insertion. Three patients mentioned mild discomfort during the insertion, while the rest did not complain. We did not experience any difficulty in draining the fluid after pleurodesis. Although all patients suffered mild to moderate pain and experienced fever after the procedure, which required medication, no early or late serious complication was observed because of pleurodesis in any patient.

The patient with malignant mesothelioma died because of sepsis due to neutropenia after 2 months. During that period, no pleural fluid aspiration was required, although minimal effusion was noted in chest X-ray. The first patient with lung cancer responded completely, and only minimal pleural effusion was noted in the 12-month follow-up. The second patient with lung cancer died 5 months after pleurodesis because of cerebral metastasis. Although some pleural effusion recurred after 3 months, no drainage was indicated. The third patient with lung cancer required another session of pleurodesis after 3 weeks, which also failed in a month. This patient underwent video-assisted thoracoscopic surgery. The fourth patient with lung cancer required another session of talc pleurodesis after 3 months. No pleural intervention was required in this patient at 8-month follow-up, while minimal effusion was noted in chest X-rays. One patient with breast cancer responded completely, and no pleural effusion was noted in the 12-month follow-up. The second patient with breast cancer had minimal pleural effusion and/or thickening for the following 9 months. The third patient with breast cancer had some pleural effusion, which did not need any intervention for the following 9 months. The fourth patient with breast cancer died because of sepsis at the third month, with some recurrent effusion, which did not need any intervention. The fifth patient with breast cancer came
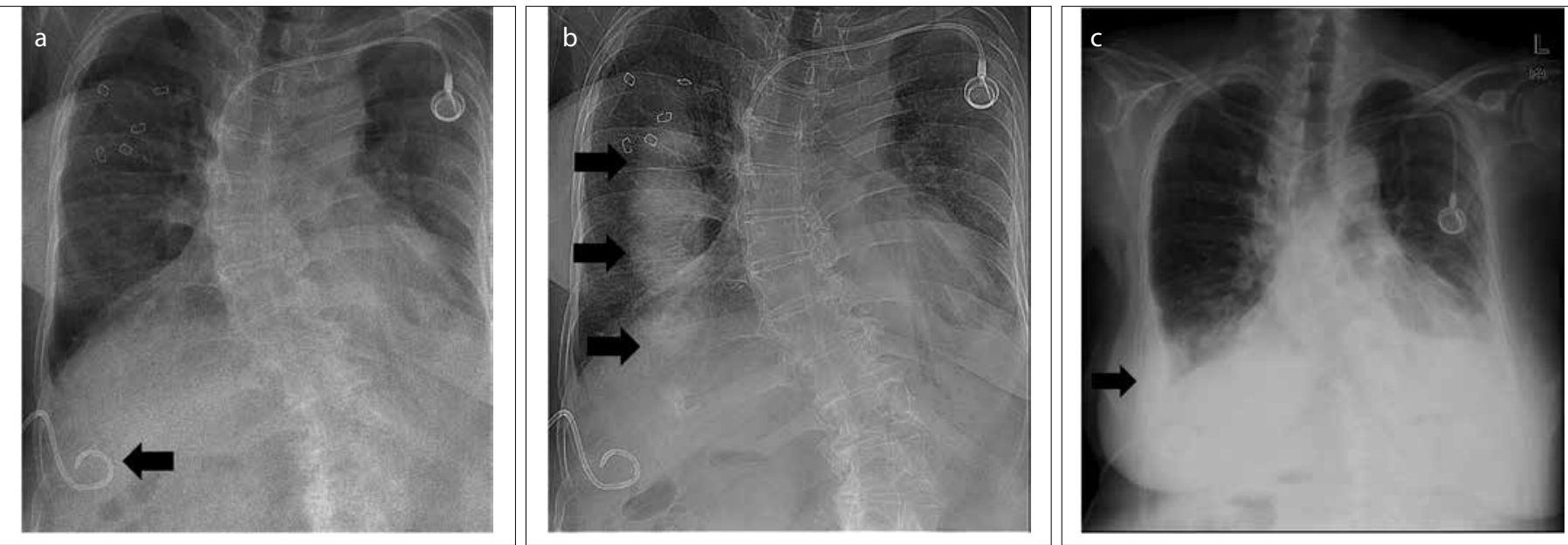

Figure 1. a-c. A 68-year-old female patient with recurrent malignant pleural effusion. Patient lying in the supine position on the fluoroscopy table. Note the pre-existing 7F pig-tail catheter on the right side (arrow). Mild left-sided pleural effusion was stable for 6 months (a). lodinated contrast medium dispersed in the pleural cavity injected via a catheter (arrows) (b). Standard chest X-ray obtained right after talc injection. Note the right-sided dense pleural fluid with talc slurry and iodinated contrast medium (arrow) (c) 
Table 1. Patient data and outcome of the pleurodesis procedures

\begin{tabular}{|c|c|c|c|c|c|}
\hline \multicolumn{2}{|l|}{ Patient } & \multicolumn{2}{|c|}{ First pleurodesis } & \multicolumn{2}{|c|}{ Second pleurodesis } \\
\hline Age (years), sex & Primary disease & Follow up & Result & Follow up & Result \\
\hline 49 , male & Malignant mesothelioma & 2 months & Complete & - & - \\
\hline 56 , male & Lung cancer, adenocarcinoma & 12 months & Complete & - & - \\
\hline 71 , male & Lung cancer, adenocarcinoma & 5 months & Partial & - & - \\
\hline 67 , male & Lung cancer, small cell & 3 weeks & Failure & 1 month & Failure \\
\hline 65 , male & Lung cancer, adenocarcinoma & 3 months & Failure & 8 months & Complete \\
\hline 64 , female & Breast cancer, invasive ductal & 12 months & Complete & - & - \\
\hline 56 , female & Breast cancer, invasive lobular & 9 months & Complete & - & - \\
\hline 68 , female & Breast cancer, invasive ductal & 9 months & Partial & - & - \\
\hline 44, female & Breast cancer, invasive ductal & 3 months & Partial & - & - \\
\hline 48 , female & Breast cancer, invasive ductal & 10 weeks & Failure & 1 month & Failure \\
\hline
\end{tabular}

back with moderate pleural effusion and mild shortness of breath in the $10^{\text {th }}$ week. When pleural fluid was recurred after 2 aspirations with a 2-week interval, talc pleurodesis was repeated. The patient came back with shortness of breath and moderate pleural effusion after 1 month, which required fluid aspiration.

We had 4 complete and 3 partial clinical success cases out of 10 patients in the first round of pleurodesis and achieved a $70 \%$ success rate. We had also 1 complete success case out of 3 patients in the second round of pleurodesis and achieved an $80 \%$ total success rate, including complete and partial ones. With regard to interventions, we had 8 successful outcomes, 5 complete and 3 partial, out of 13 patients with pleurodesis (62\%). Patient data and outcomes of the procedures are summarized at Table 1 . Out of 8 patients with complete or partial successful pleurodesis, 7 mentioned moderate to high relief in dyspnea, while 1 patient mentioned minimal relief.

\section{DISCUSSION}

Malignant pleural effusion is a common complication of advanced malignancy $(1,2)$. The most common causes for MPE are lung cancer, breast cancer, lymphoma, ovarian cancer, and gastric cancer (1). Malignant mesothelioma, a very rare disease, may also present with large pleural effusion at the time of diagnosis (1). Dyspnea, cough, and chest pain are the primary symptoms of MPE (2). Even in patients respond to systemic treatment, local therapy for recurrent MPE may still be required (2).

Pleurodesis is defined as the insertion of a sclerosing agent into the pleural cavity to induce inflammation with the resulting adhesion of pleural layers. Although tetracycline, bleomycin, and iodopovidone have been used in pleurodesis in different success and complication rates, talc pleurodesis is a widely accepted and effective procedure to treat MPE (2-10). Sterile talc with an average particle size of $25 \mu \mathrm{m}$ can be safely used in pleurodesis (11). Intrapleural administration of talc can be performed via videothoracoscopy or a chest tube, and this requires hospital admission $(6,7)$. However, pleurodesis via a percutaneously inserted $12-18 \mathrm{~F}$ pig-tail catheter could be performed with-hout hospital admission $(4,5)$.

Possible adverse events commonly observed in patients with talc pleurodesis include the incidence of fever and pain (2). All of our pa tients suffered mild to moderate pain and experienced fever after the procedure, which was easily controlled with medication. Although talc pleurodesis has been considered a somewhat safe intervention, complications such as acute pneumonitis, empyema, wound infection, and even acute respiratory failure may be observed in the follow up of pleurodesis (2). We did not observe any early or late serious complications.

In this study, we present 13 talc pleurodesis procedures successfully performed via 7F or 8F catheters in 10 patients with recurrent MPE. These very-small-bore catheters never need dilation of the tract and are easily inserted as a single-step procedure. Width of the holes of the catheter was wide enough to inject the talc slurry effectively and to drain the fluid after the procedure. The $8 \mathrm{~F}$ catheters were proven to be safe and effective in the treatment of pleural effusion, but, to the best of our knowledge, they have not been used in talc pleurodesis previously (12).

The major limitation of this study is the limited number of patients. Our total complete and partial clinical success rates, 8 out of 10 patients ( $80 \%)$ and 8 out of 13 interventions (62\%), respectively, were similar to those reported previously, although we had a limited number of patients and the time of follow-up differed significantly $(2,3)$.

\section{CONCLUSION}

In conclusion, talc pleurodesis through 7F or 8F catheters may be performed in selected patients in the treatment of recurrent MPE. In comparison with $12-18 \mathrm{~F}$ catheters, $7 \mathrm{~F}$ or $8 \mathrm{~F}$ catheters may be favored because of reduced discomfort and ease of insertion with similar efficacy. Further comparative studies, possibly with more subjects, are required to reveal the exact efficacy of this method.

Ethics Committee Approval: Ethics committee approval was received for this study from the ethics committee of Yeditepe University.

Informed Consent: Written informed consent was obtained from patients who participated in this study.

Peer-review: Externally peer-reviewed.

Conflict of Interest: No conflict of interest was declared by the author.

Financial Disclosure: The author declared that this study has received no financial support. 


\section{REFERENCES}

1. Egan AM, McPhillips D, Sarkar S, Breen DP. Malignant pleural effusion. Q J Med 2014; 107: 179-84. [CrossRef]

2. Xia H, Wang XJ, Zhou Q, Shi HZ, Tong ZH. Efficacy and safety of talc pleurodesis for malignant pleural effusion: A meta-analysis. PLoS One 2014; 9: e87060. [CrossRef]

3. Srour N, Amjadi K, Forster A, Aaron SH. Management of malignant pleural effusions with indwelling pleural catheters or talc pleurodesis. Can Respir J 2013; 20: 106-10. [CrossRef]

4. Özkul S, Turna A, Demirkaya A, Aksoy B, Kaynak K. Rapid pleurodesis is an outpatient alternative in patients with malignant pleural effusions: a prospective randomized controlled trial. J Thoracic Dis 2014; 6: 1731-5.

5. Terra RM, Teixeira LR, Bibas BJ, Pego-Fernandes PM, Vargas FS, Jatene FB. Effectiveness and safety of outpatient pleurodesis in patients with recurrent malignant pleural effusion and low performance status. Clinics (Sao Paulo) 2011; 66: 211-6. [CrossRef]

6. Bhatnagar R, Laskawiec-Szkonter M, Piotrowska HE, Kahan BC, Hooper $\mathrm{CE}$, Davies $\mathrm{HE}$, et al. Evaluating the efficacy of thoracoscopy and talc poudrage versus pleurodesis using talc slurry (TAPPS trial): pro- tocol of an open-label randomised controlled trial. BMJ Open 2014; 4: e007045. [CrossRef]

7. Gawron G, Gabryś J, Barczyk A. Talc slurry pleurodesis via chest tube in department of pulmonology - a 24-case study. Pneumonol Alergol Pol 2013; 81: 439-47.

8. Agarwal R, Khan A, Aggarwal AN, Gupta D. Efficacy \& safety of iodopovidone pleurodesis: a systematic review \& meta-analysis. Indian J Med Res 2012; 135: 297-304.

9. Ibrahim IM, Dokhan AL, El-Sessy AA, Eltaweel MF. Povidone-iodine pleurodesis versus talc pleurodesis in preventing recurrence of malignant pleural effusion. J Cardiothorac Surg 2015; 10: 64. [CrossRef]

10. Fysh ETH, Thomas R, Read CA, Lam BCH, Yap E, Horwood FC, et al. Protocol of the Australasian Malignant Pleural Effusion (AMPLE) trial: a multicentre randomised study comparing indwelling pleural catheter versus talc pleurodesis. BMJ Open 2014; 4: e006757. [CrossRef]

11. Janssen JP, Collier G, Astoul P, Tassi GF, Noppen M, Rodriguez-Panadero F, et al. Safety of pleurodesis with talc poudrage in malignant pleural effusion: a prospective cohort study. Lancet 2007; 369: 1535-9. [CrossRef]

12. Gammie JS, Banks MC, Fuhrman CR, Pham SM, Griffith BR, Keenan RJ, et al. The pigtail catheter for pleural drainage: a less invasive alternative to tube thoracostomy. JSLS 1999; 3: 57-61. 